\title{
Closed Loop RF Management Algorithm for Enterprise High Density WLANs
}

\author{
Rohit Gupta, Tommy Shing, Sumit Roy \\ Dept. of Electrical Engineering, University of Washington \\ Seattle, WA 98195 \\ \{rohitg, tshing, sroy\}@u.washington.edu
}

\author{
Jing Zhu \\ Communication Technology Lab, Intel Corporation \\ Hillsboro, OR 97124 \\ jing.z.zhu@intel.com
}

\begin{abstract}
The growing adoption of 802.11 networks in the enterprise segment has led to the emergence of High Density (HD) WLAN scenarios where large (100-1000) numbers of clients are serviced by 10-100s of APs in a multi-cell environment. This leads to an interference limited environment due to limited spectrum availability; hence network design for throughput scalability becomes the primary design challenge. Current industry practice leans towards centralized RF management of HDWLAN Enterprise deployments using a RF controller. The main focus of the work in this paper is a new CS/RT (Carrier Sense/Receiver threshold) adaptation algorithm in which APs, Clients and RF controllers participate collaboratively to mitigate the interference. Performance comparisons of the proposed CS/RT adaptation compared with current rate adaptation algorithms such as SampleRate [2] and ARF [1] are presented.
\end{abstract}

\section{INTRODUCTION}

The rapid growth in mobile client handheld computingcum-communication devices with embedded 802.11 radios has contributed to the proliferation of 802.11 Wireless LAN (WLAN) networks in diverse environments beginning with the home, spreading to public hotspots and now, the enterprise. In the enterprise segment (and also possibly in public arenas with multiple WLAN providers with their own separate network domains that are un-coordinated), the inter-AP separations can be as small as a few meters [13], whereby co-channel interference is the dominant impairment. Traditionally, 802.11 networks for isolated single hot-spots (such as the individual home) have been designed based on RF site-survey for coverage, whereby a minimum received signal strength (RSSI) and hence a minimum link rate per end user is provisioned over the desired region. This approach is inappropriate for High Density (HD) WLAN networks where providing appropriate quality of service (bandwidth, delay, packet loss) guarantees to all end users is the primary concern.

RF management is increasingly becoming a dynamic and challenging task, which is expected to provide efficient and effective interference mitigation capability and scale network capacity with user density. The network management architecture requires a balance between the completely centralized (significant communication resources spent on communicating client network status for enhanced algorithms), and completely decentralized (unable to meet performance criteria without some coordination).

Recent developments in IEEE $802.11 \mathrm{k} / 802.11 \mathrm{v}$ favor a centralized RF controller: clients collect local statistics that are communicated to the controller; in return, various parameters of AP and clients are determined by the RF controller. In the above context, the main distinguishing contributions of our approach include: a) A closed loop SNIR (Signal to Noise + Interference Ratio) based algorithm for CS/RT adaptation so as to manage interference in multiple cells of HDWLAN.

b) Performance evaluation of proposed SNIR adaptation with current rate adaptation algorithms like SampleRate [2] and ARF [1] in this framework. We also propose guidelines that RF controllers should use in adapting "global" minimum SNIR threshold in the presence of local rate adaptation algorithms.

\section{RELATED WORK}

Recently, more and more research efforts [3]-[12] have been dedicated to leveraging spatial reuse to improve network capacity of HD-WLAN. It has been shown in [4][5] by Zhu et. al that CCA adaptation can dramatically improve the performance of WLANs. Yang et al. [7] then proposed an analytical model to address the impact of MAC overhead on the optimal CCA threshold, considering both bandwidth independent overhead from PHY header, slot time, etc., and bandwidth dependent overhead caused mainly by collisions. Moreover, Zhan et al. [9] studied the impact of data rates, multi-hop forwarding, node topology, hidden/exposed terminal problems, and bidirectional handshakes on determining the optimal carrier sensing range. The CCA threshold adaptation algorithm in [11][9] suggest setting the fixed CCA threshold in an entire network; however, we contend that setting a variable CCA threshold at each node according to local network conditions will better maximize spatial reuse of the network as cells have different density and traffic patterns. The analytical approach of [10] provides the most concrete guidance for the optimal CCA threshold for single rate networks: setting the threshold so that the interference range equals the carrier sense range.

In [3], the authors propose a CCA adaptation algorithm called ECHOS, which uses a measurement-driven approach for tuning the CCA threshold of APs and clients. The clients report the measured interference levels to their respective APs. Each AP, if possible, tries to set its CCA threshold so as to support the highest data rate at the farthest client. However, it has been shown in [11] that supporting the highest data rate is not necessarily an optimum setting in high density networks. The results presented in OPNET simulations of our algorithm in Section V corroborate this observation. Recently, authors in [13] propose interference mitigation through transmit power control. However, power control is a totally separate problem, 
so in this paper we focus mainly on CCA based adaptation algorithms.

There has also been testbed implementations [6][8] regarding end-to-end (E2E) CCA adaptation algorithm based on the maximum PER measurement in [8] for single rate networks, and prototyped using Intel Centrino ${ }^{\mathrm{TM}}$ radio. However, the algorithm for CCA self-adaptation in [8] starvation in some links (a.k.a. the fairness problem). The above algorithms also have a limitation of dynamically setting CCA threshold for multi-rate, multi-cell networks and there have been very few studies on the effect of dynamically setting CCA/RS threshold in current rate adaptation algorithms. Our current work tries to bridge this gap by making use of RSSI measurements at the APs and CLs and driving the adaptation of the CCA threshold according to the current RF conditions to solve capacity problems in HDWLAN. We also discuss the impact of rate adaptation algorithms such as SampleRate [2] and ARF [1] on the performance of the proposed CCA/RS adaptation algorithm.

\section{ClOSED LOOP AdAPTATION ALGORITHM FRAMEWORK FOR HDWLANS}

In the light of above discussion, the proposed framework is such that the RF management controller lays down rules to control the client behavior by collecting statistics using IEEE $802.11 \mathrm{k}$ standard. It should be noted that the RF controller does NOT control/micromanage the client algorithm behavior, but, it nevertheless enforces some high-level performance objectives which should be met by all the clients on the network. The objective of this research is to find out what these key high level objectives are and suggest distributed algorithms to tune wireless MAC parameters which can be implemented at APs and clients to meet the performance objectives set by the network.

The proposed rule which is enforced by the RF controller is Minimum SNIR threshold $\left(S_{\min }\right)$. This rule and its associated algorithm is described in detail in section IV and the main objective of this rule is to determine the optimal PHY layer worst case minimum SNIR (Signal to Noise plus Interference ratio) criteria which should be satisfied at all times by all the nodes in the network. $S_{\min }$ is broadcasted by the RF controller to satisfy the minimum PHY rate in the network (which in case of $802.11 \mathrm{a}$ is $6 \mathrm{Mbps}$ ). The research aims at evaluating network performance by varying this threshold. Note, here that the clients closer to their respective APs might experience SNIR on "average", so they might use higher PHY rate as directed by their rate adaptation algorithm.

\section{CS/RT Threshold AdAPTATION USING MinimuM SNIR $\left(S_{M I N}\right)$ THRESHOLD}

Before describing the algorithm, we would like to clarify several definitions used in the paper:

Clear Channel Assessment/Carrier Sensing (CCA/CS) threshold: This threshold describes the behavior of transceiver in deciding whether the wireless channel is busy. If the sampled RF energy is above the $C S / C C A$ threshold, then the channel is assumed to be busy. The default setting of this threshold in current hardware is fixed at around $-95 \mathrm{dBm}$.

Receiver Sensitivity $(R S)$ : Receiver Sensitivity refers to the minimum power of the desired signal at the receiver that is required for the successful decoding of the signal in the presence of noise alone. The Receiver Sensitivity depends on the modulation and coding scheme and is dependent on the hardware implementation.

Receiver Threshold (RT): The Receiver threshold defines that value of received power below which the receiver circuitry will not attempt to decode the received signal. By default, the Receiver threshold and the $C C A$ threshold are set to be approximately equal to, and slightly higher than the noise power. The default setting of this threshold in current hardware is fixed at around $-95 \mathrm{dBm}$. The Receiver Threshold for each node in a particular cell is adapted to include all APs/clients in that cell so as to include hidden terminals within same cell and also avoid stronger last collisions [8].

Notice that the $R S$ and $R T$ are altogether different quantities. While the former is determined and fixed by the hardware capabilities, the latter is configurable. The main goal for $C S / R T$ adaptation is to make sure that $C S / R T$ settings satisfy the $S_{\min }$ constraints set by the centralized RF controller.

\section{A. Assumptions}

1) All clients and APs in the network lower their Carrier Sense/Receiver Threshold (CS/RT) threshold and receiver sensitivity threshold periodically at the same time to a minimum level $(\sim-95 \mathrm{dBm})$ to make measurements regarding the signal strength from all of their neighbors. All clients also send small beacon packets during this measurement interval to facilitate signal strength measurements by their neighbors. Reducing $C S / R T$ threshold to the lowest value during measurement interval ensures that the $C S / R T$ range is increased to the maximum; therefore, only one node in the $C S$ range will be able to transmit beacon packets and all nodes can accurately measure received power from their neighbors. These neighbors may be from the same or adjoining cells. We assume that lowering of $C S / R T$ threshold to $-95 \mathrm{dBm}$ ensures that "only" one node in its $C S / R T$ range transmits a beacon measurement packet while the rest of the nodes make power measurement. However, it is possible, though unlikely, for different nodes in same $C S / R T$ range to transmit beacon packet at the same time, thus corrupting the measurement. To make the measurements more accurate, we will take an average over sufficient number of measurements over a time window. It should be noted that the duration of measurement interval and the invocation of measurements are directed by the RF management server.

2) All nodes are trying to achieve the constraint broadcasted by the RF controller, $S_{\min }$.

\section{B. Algorithm Description}

Figure $2 \mathrm{a}$ is the flow chart demonstrating the workings of this algorithm. The algorithm is initiated at each of the individual APs and clients ONLY after each of the nodes have 
information regarding the average received powers from other neighboring APs and clients from inside and outside its cell (as described below). $S_{\min }$ is also broadcasted by the centralized RF controller before the algorithm is initiated.

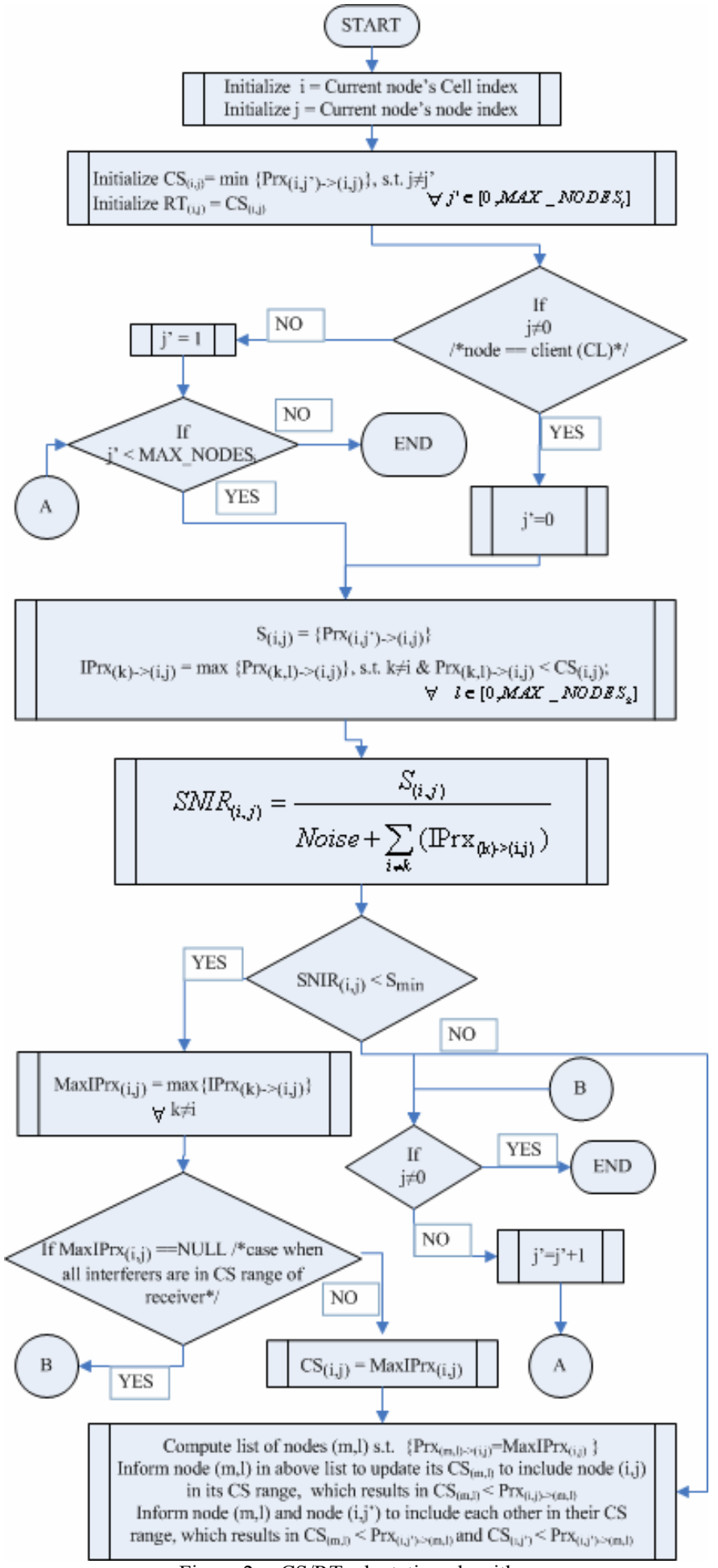

Figure 2a. CS/RT adaptation algorithm
Each node in the network is indexed by subscript $(i, j)$, where " $i$ " refers to the cell index, and " $j$ " refers to the node index. All APs have their "node index" set to " 0 ", and all clients have a non-zero node index.

It should be noted in figure $2 \mathrm{a}$ that each node informs its interferer if $S_{\min }$ is not satisfied, so that the potential "interferer" can include the node in its $C S$ range. It is beyond the scope of this algorithm on how certain receiver node informs a potential interferer to reduce its $C S$ threshold. All nodes, for example can take assistance from centralized RF controller to inform potential interferer to reduce its $C S$ threshold.

TABLE I ALGORITHM PARAMETERS

\begin{tabular}{|c|c|}
\hline $\mathrm{CS}_{(\mathrm{i}, \mathrm{j})}, \mathrm{j} \neq 0$ & CS threshold of client "j" at cell "i" \\
\hline $\mathrm{RT}_{(\mathrm{i}, \mathrm{j})}, \mathrm{j} \neq 0$ & Receiver threshold of client "j" in cell "i" \\
\hline $\mathrm{CS}_{(\mathrm{i}, 0)}$ & CS threshold of AP in cell "i"" \\
\hline $\mathrm{RT}_{(\mathrm{i}, 0)}$ & Receiver threshold at the AP in cell "i" \\
\hline $\operatorname{Prx}_{(\mathrm{k}, \mathrm{l})->(\mathrm{i}, \mathrm{j})}$ & $\begin{array}{l}\text { Received power from node }(k, 1) \text { measured at } \\
\text { node }(i, j)\end{array}$ \\
\hline $\operatorname{IPrx}_{(\mathrm{k})->(\mathrm{i}, \mathrm{j})}$ & $\begin{array}{l}\text { Worst case interference power from cell " } k \text { " } \\
\text { incident on node }(i, j)\end{array}$ \\
\hline$S_{\min }$ & $\begin{array}{l}\text { SNIR minimum threshold broadcasted by the } \\
\text { centralized RF controller }\end{array}$ \\
\hline MAX_NODES & $\begin{array}{l}\text { Maximum number of nodes including AP and } \\
\text { clients in cell "i" }\end{array}$ \\
\hline MAX_CELLS & Maximum number of cells in the current network. \\
\hline
\end{tabular}

At the end of the SNIR adaptation algorithm we find that there are some cases in which the $C S$ range of say node $\mathrm{A}$ include node $\mathrm{B}$, but node B's $C S$ range does not include node A. If this case is left unattended, then node B may try to keep sending data to its intended receiver without deferring to node A, resulting in starvation of throughput for node A. To avoid starvation problems like the aforementioned, we need to run the CS balance algorithm, which makes sure that all nodes are in the $C S$ range of particular node, e.g. node $\mathrm{A}$, also include node $\mathrm{A}$ into their respective $\mathrm{CS}$ ranges. The flow chart for CS balance algorithm is given in figure $2 \mathrm{~b}$.

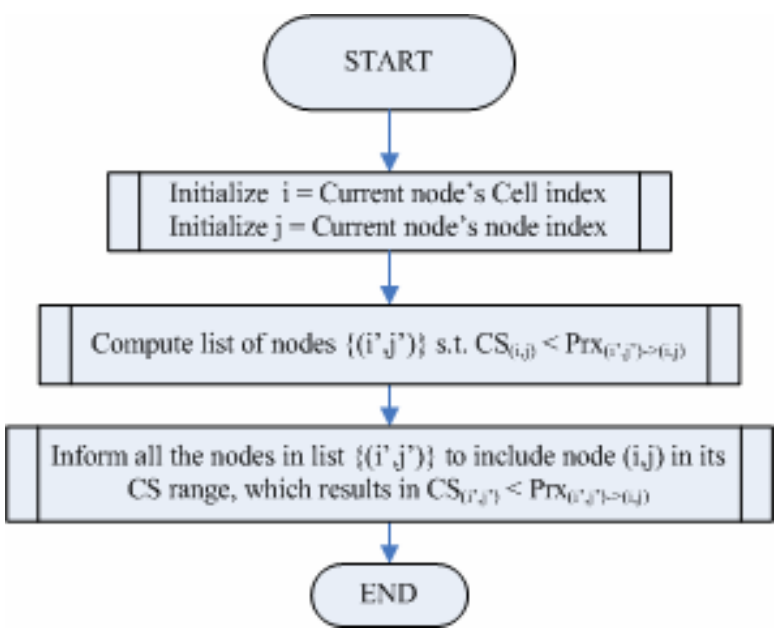

Figure 2b. Flow chart for CS balance algorithm 


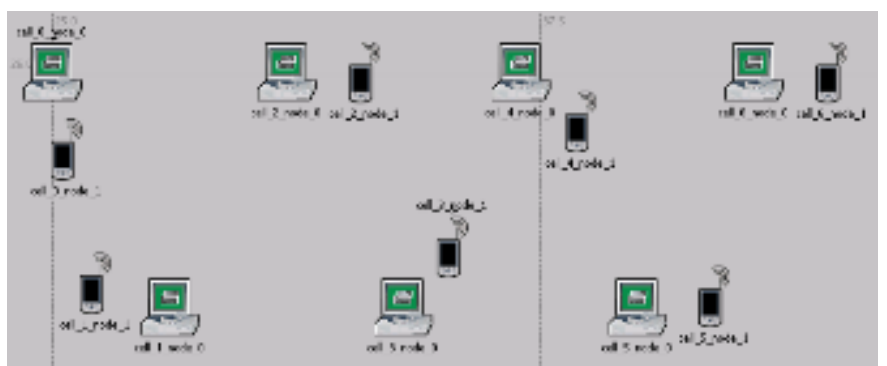

Figure 3. Network topology

\section{OPNET Experiment Simulation:}

We conducted OPNET simulation using the algorithm mentioned above on the HD WLAN topology indicated in figure 3. All nodes with node index equal to " 0 " are APs and all the traffic in each of the conducted experiments is saturated uplink UDP traffic with exponentially distributed packet interarrival times. All APs are in a grid with horizontal and vertical distance of $6 \mathrm{~m}$. Clients are uniformly distributed by angle with a radius of $2 \mathrm{~m}$ from their respective AP.

\section{Simulation Results For CCA AdAPtation ALGORITHM USING $\mathrm{S}_{\text {MIN }}$}

We now describe the simulation results for a scenario in figure 3 showing the performance improvement achievable using various values of $S_{m i n}$. We are using OPNET v11.0 and implemented ARF [1] and SampleRate [2] adaptation algorithm in OPNET. The UDP traffic is generated only from clients to their respective APs and all nodes are operating in saturated condition. All nodes use a fixed IEEE 802.11a PHY layer with transmit power of $0.1 \mathrm{~W}$ and fixed packet size of 1000 bytes. We have implemented the SNIR adaptation algorithm in a centralized manner in OPNET to ease the implementation as it is easier to calculate received power from the neighbors according to the path loss model and distance between nodes. Hence, before the start of the simulation the algorithm is run for each scenario which sets the CS/RT threshold for each node in the network.

Table 2 lists the SNIR requirement $\left(\mathrm{S}_{0}\right)$ of different IEEE 802.11a rates. Recall that $S_{\min }$ is set to satisfy minimum PHY rate in the network. Hence, if the minimum PHY rate to be satisfied is $12 \mathrm{Mbps}$, then $S_{\min }$ should be greater than $\mathrm{S}_{0}$. As it can be seen from table 2, $S_{\min }$ used in our simulations is slightly higher $(0.3 \mathrm{~dB})$ than $\mathrm{S}_{0}$ for a particular PHY rate.

TABLE II

IEEE 802.11A PHY RATE AND SNIR THRESHOLD REQUIREMENT

\begin{tabular}{|c|c|c|c|c|c|c|c|c|}
\hline Rate $(\mathrm{Mbps})$ & 6 & 9 & 12 & 18 & 24 & 36 & 48 & 54 \\
\hline $\mathrm{S}_{0}(\mathrm{~dB})$ & 4.5 & 6.3 & 7.5 & 9.3 & 15.0 & 16.8 & 21.6 & 22.3 \\
\hline $\operatorname{Smin}(\mathrm{dB})$ & 4.8 & 6.6 & 7.8 & 9.6 & 15.3 & 17.1 & 21.9 & 22.6 \\
\hline
\end{tabular}

\section{A. Simulation Results for Fixed Rate Multi-Cell Networks}

We now discuss the simulation results for fixed rate multicell high density WLAN network using SNIR adaptation algorithm. We compare these results against fixed CS/RT threshold of $-95 \mathrm{dBm}$ in current legacy hardware. We ran the SNIR adaptation algorithm discussed above which sets the appropriate $\mathrm{CS} / \mathrm{RT}$ thresholds for all the nodes and then the uplink UDP saturated traffic is generated from clients to their respective APs with fixed rate corresponding to the particular $S_{\text {min }}$ from table 2.

TABLE III

TYPICAL CS/RT SETTINGS, PER, AND THROUGHPUT FOR $S_{M I N}=9.6 \mathrm{~dB}$

\begin{tabular}{|c|c|c|c|c|}
\hline & \multicolumn{4}{|c|}{$S_{\min }=9.6 \mathrm{~dB}$, PHY rate $=18$ Mbps } \\
\hline $\begin{array}{c}\text { Node } \\
\text { index } \\
(\mathrm{I}, \mathrm{j})\end{array}$ & $\begin{array}{c}\mathrm{CS}_{(\mathrm{i}, \mathrm{j})} \\
(\mathrm{dBm})\end{array}$ & $\begin{array}{c}\mathrm{RT}_{(\mathrm{i}, \mathrm{j})} \\
(\mathrm{dBm})\end{array}$ & PER & $\begin{array}{c}\text { Throughput } \\
(\mathrm{Mbps})\end{array}$ \\
\hline$(0,0)$ & -53.82 & -35.76 & & 6.23 \\
\hline$(0,1)$ & -53.66 & -35.76 & 0.0027 & \\
\hline$(1,0)$ & -53.51 & -35.71 & & 7.76 \\
\hline$(1,1)$ & -48.97 & -35.71 & 0.0028 & \\
\hline$(2,0)$ & -51.15 & -35.75 & & 2.02 \\
\hline$(2,1)$ & -53.82 & -35.75 & 0.0041 & \\
\hline$(3,0)$ & -51.53 & -35.74 & & 8.03 \\
\hline$(3,1)$ & -52.85 & -35.74 & 0.0025 & \\
\hline$(4,0)$ & -53.13 & -35.74 & & 1.75 \\
\hline$(4,1)$ & -51.26 & -35.74 & 0.0358 & \\
\hline$(5,0)$ & -51.53 & -35.75 & & 2.22 \\
\hline$(5,1)$ & -53.13 & -35.75 & 0.0048 & \\
\hline$(6,0)$ & -51.53 & -35.75 & & 10.86 \\
\hline$(6,1)$ & -51.05 & -35.75 & 0.0025 & \\
\hline
\end{tabular}

Table 3 lists the $C S / R T$ parameters resulting from the $S_{\text {min }}$ adaptation algorithm for all nodes and cells along with individual packet error rate (PER) and throughput for $S_{\min }=9.6$ dB. The fixed PHY rate-18 Mbps-for the whole network for a particular $S_{\min }$ is the one which satisfies $\mathrm{S}_{0}$ requirement from table 2. It should be noted from table 3 , that there is appreciable packet loss due to the collisions from nodes within same cell. SNIR adaptation algorithm is intended to reduce the packet loss due to interferers "outside" the CS range of particular node, and it is of little help in preventing packet loss due to collisions within same CS range. It should also be noted that some nodes get more throughput than others because they experience less packet loss, have more chances to access the channel, or both, depending on the network topology. In this particular case, cells 2, 4, and 5 have less throughput because they include more nodes compared to other cells. However, it should be noted that SNIR adaptation outperforms fixed $C S / R T$ settings of $-95 \mathrm{dBm}$. The important question that needs to be answered is how should the RF controller decide on a particular $S_{\min }$, or whether different $S_{\min }$ can be used for different cells to maximize throughput? The answer to this design question is discussed in some detail in the later sections. We now analyze the worst case packet loss for various $S_{\min }$ thresholds along with aggregate throughput. Worst case packet loss at a particular $S_{\min }$ is defined as maximum PER (PERmax) experienced by any cell for a particular $S_{\min }$ threshold. The plots of $S_{\min }$ Vs PERmax and aggregate throughput are given in figure 4 . We also plot the aggregate throughput and PERmax for the "legacy" case, in which CS/RT threshold is fixed at $-95 \mathrm{dBm}$. It is important to note that all the $S_{\min }$ parameters outperform the "legacy" MAC settings. Another important feature to note about $S_{\min }$ adaptation algorithm is that for fixed rates, PERmax increases with higher $S_{\min }$. The reason for this is that higher rates are used for higher $S_{\min }$, and have less tolerance for collisions. 
Surprisingly, for the scenario in consideration, the aggregate throughput is higher for higher $S_{\min }$ despite more collisions experienced by higher PHY rates.

\section{B. Simulation results for SNIR adaptation algorithm with rate adaptation algorithms:}

We now describe the performance of CS/RT adaptation algorithm with the current rate adaptation algorithms: SampleRate [2] and ARF [1].

SampleRate [2] is transmitter based rate adaptation algorithm which starts with the highest bit rate. It measures the number of retries for each rate and tries to minimize the average transmission for sending a packet. For this purpose, it keeps track of average transmission time for different rates used in the past and uses the rate which delivers the minimum average transmission time. SampleRate does not use a particular rate if it experiences 4 retries for that rate and it tries a new rate every $10^{\text {th }}$ packet which could possibly deliver better throughput. SampleRate assumes that channel conditions change every ten seconds and hence flushes the history of retry count for all rates if they are older than 10 seconds.

ARF [1] is also a transmitter based algorithm and much simpler than SampleRate w.r.t. implementation. Given the number of retries that a transmission used and whether or not it was successfully acknowledged, ARF adjusts the bit-rate for the destination based on the following criteria:

1) Move to the next lowest bit-rate if the packet was unacknowledged consecutively.

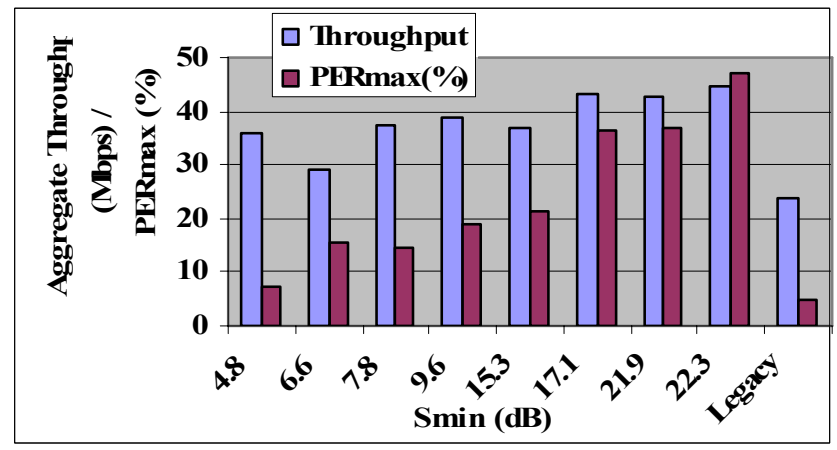

Figure 4. Smin Vs Aggregate throughput and PERmax

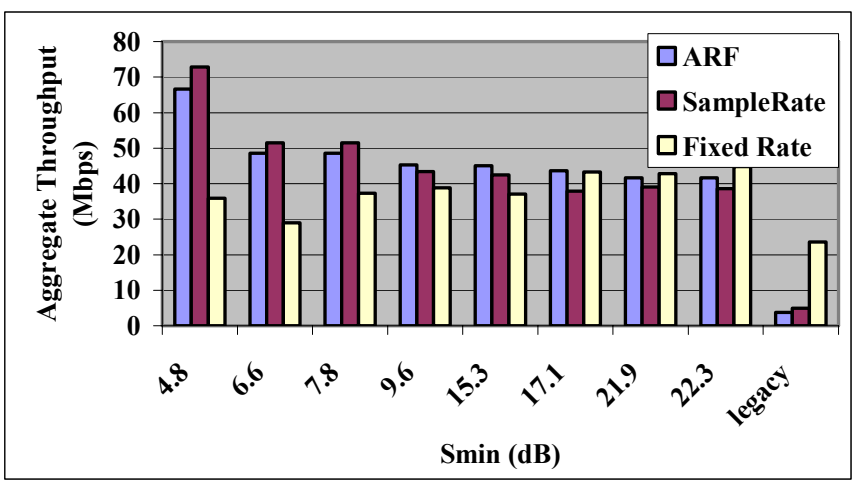

Figure 5. $S_{\min }$ Vs Aggregate throughput for rate adaptation and fixed rate
2) Move to the next highest bit-rate if 10 successive transmissions have occurred without any retransmissions.

3) Otherwise, continue at the current bit-rate.

We now describe the results of $C S / R T$ adaptation along with SampleRate and ARF. Figure 5 gives a plot aggregate throughput for $S_{\min }$ Vs different rate adaptation algorithms. We also plot the "legacy" case in this graph which indicates the throughput for default fixed $C S / R T$ settings of $-95 \mathrm{dBm}$. It is instructive to note that at lower $S_{\text {min }}$ regimes, say $4.8 \mathrm{~dB}$, SampleRate outperforms ARF because ARF does not select best rates in lossy channels. The similar behavior has also been observed in [2].

However, it is interesting to note that in higher $S_{\min }$ regime, ARF slightly outperformed SampleRate. To explain this behavior, we plotted the individual throughput/PHY rate and number of retries Vs time at cell 0 for $S_{\text {min }}=22.3 \mathrm{~dB}$ in figure $6 \mathrm{a}, 6 \mathrm{~b}$ and $6 \mathrm{c}$ respectively. We notice that initially SampleRate uses both rates $54 \mathrm{Mbps}$ and $48 \mathrm{Mbps}$ until around $1.9 \mathrm{sec}$, when it stops using $54 \mathrm{Mbps}$ because it experiences 4 retries at $54 \mathrm{Mbps}$ at $1.9 \mathrm{sec}$. It can be seen that throughput drops from around $10 \mathrm{Mbps}$ to $8 \mathrm{Mbps}$ because SampleRate is not using 54Mbps anymore. Again at $4.47 \mathrm{sec}$, it experiences 4 retries at $48 \mathrm{Mbps}$ and drops down the rate again from $48 \mathrm{Mbps}$ to $36 \mathrm{Mbps}$. However, this time there is not appreciable drop in throughput. Again at 11.8 seconds which is around 10 seconds after first event at $1.9 \mathrm{sec}$, cell 0 again starts using $54 \mathrm{Mbps}$ as old entries are flushed out after 10 seconds. This results in choosing more conservative rates, which perform poorly in terms of throughput as it can be seen from the figure of $6 \mathrm{a}$ that choosing the PHY rate of $54 \mathrm{Mbps}$ is the best choice notwithstanding occasionally experiencing 4 retries. This results in variations of throughput, which in this case is around $3 \mathrm{Mbps}$ and causes SampleRate to perform poorly compared to both ARF and a fixed rate of $54 \mathrm{Mbps}$. We plan to make modifications to SampleRate and develop a more efficient PER based rate adaptation algorithm, which selects the rate based on both "retry count" and PER to compare which rate maximizes throughput. Due to the lack of space, we are not putting the performance results of ARF. However, we observe that for low $S_{\text {min }}$ ARF is more inefficient than SampleRate in selecting the rate that give maximum throughput and it also waste more time in retrying rates that didn't work most of the time. Similar conclusions regarding ARF's performance have been observed by Bicket et. al in [2].

We also ran our $S_{\min }$ adaptation along with rate adaptation algorithm for various high density topologies. For example, topology $\left\{x^{*} y\right\}$ indicates that APs are organized in a grid of $\left\{x^{*} y\right\}$ with Inter-AP distance of $6 \mathrm{~m}$ and clients are randomly distributed within a radius of $2 \mathrm{~m}$. The results for aggregate throughput for all of these scenarios are plotted in figure 7 . The results in figure 7 and figure 5 clearly suggest that setting $S_{\text {min }}$ to be $4.8 \mathrm{~dB}$, which is to just support minimum PHY rate of the network is a good design choice in the presence of rate adaptation algorithms. However, when fixed rates are being used in the network, then $S_{\text {min }}$ that gives best performance can vary according to the network topology. 


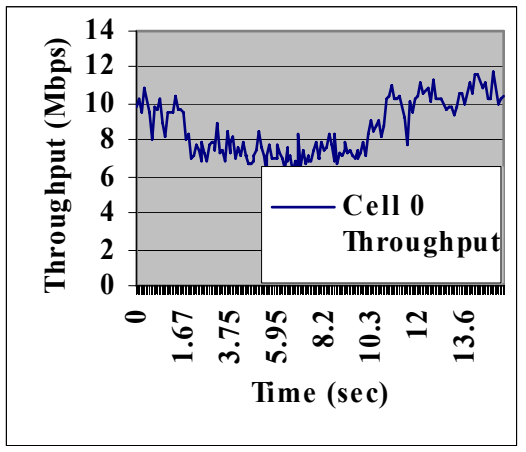

Figure 6a: Plot of Throughput Vs time at $S_{\min }=22.3 \mathrm{~dB}$ for SampleRate algorithm

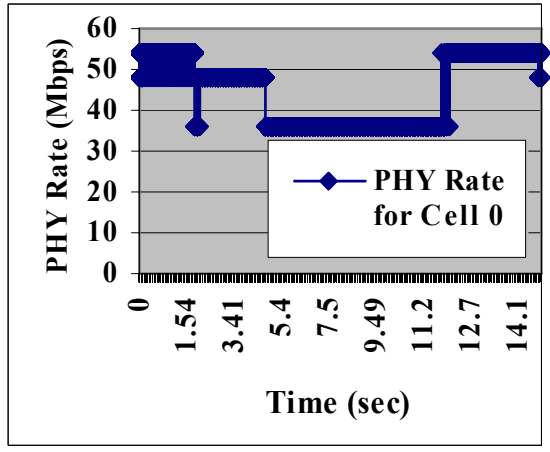

Figure 6b: Plot of PHY Rate Vs time at $S_{\min }=22.3 \mathrm{~dB}$ for SampleRate algorithm

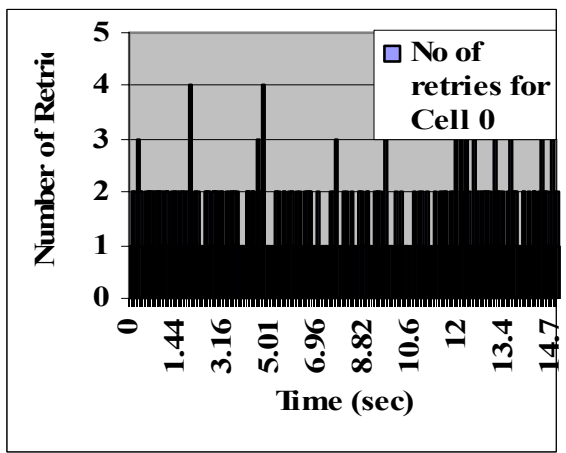

Figure 6c: Plot of Retries Vs time at $S_{m i n}=22.3$ dB for SampleRate algorithm

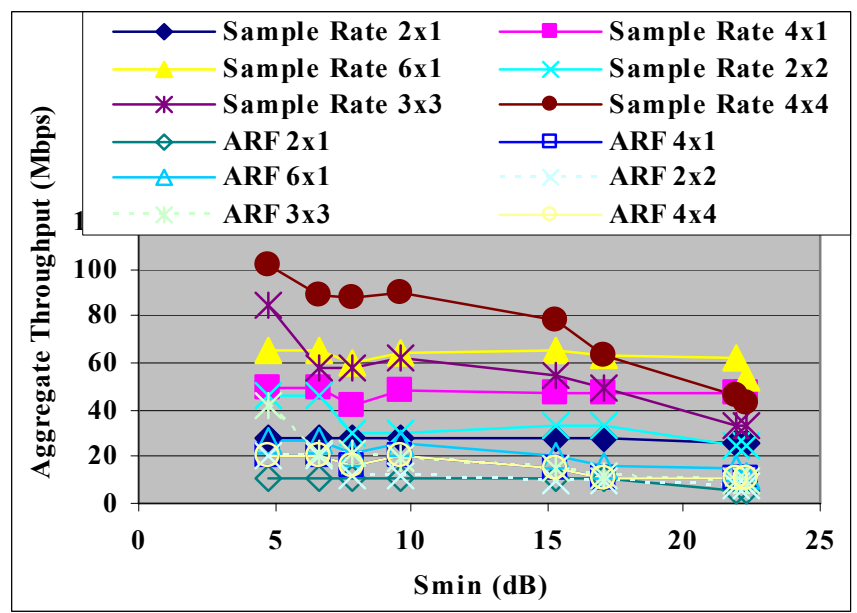

Figure 7: Plot of Aggregate throughput for Sample Rate and ARF for various high density configurations

The reason that $S_{\min }$ gives better performance in presence of rate adaptation algorithm is that setting lower $S_{\min }$ results in higher spatial reuse of the network and also reduces the collision domain of each node, however the downside is that each node has to use a lower rate and rate adaptation has to be adaptive enough to select the rate that gives best performance in terms of throughput. We also observed that the rate that gives best performance in terms of throughput incurs some amount of losses.

\section{CONCLUSiOns/Future Directions}

In conclusion, we propose a $C S / R T$ based adaptation algorithm based on $S_{\min }$, which can be easily implemented in a distributed way using a RF management server. We also establish that setting $S_{\min }$ to be of lowest value that supports minimum PHY rate in the network is a good design choice in the "presence of rate adaptation algorithms". However, setting $S_{\min }$ to be of lowest value might not maximize the aggregate throughput of the fixed rate network.

We also demonstrate the performance of $C S / R T$ based adaptation in the presence of rate adaptation algorithms such as ARF and SampleRate. We also point out the shortcomings of the SampleRate algorithm in discarding the optimal rates based on "retry" count only.
We plan to extend the RF management framework to include fairness objectives which can be set by RF controller and implemented on all the nodes in a distributed manner using other MAC parameters in the future.

\section{REFERENCES}

[1] A. Kamerman and L. Monteban. WaveLAN-II: A high-performance wireless LAN for the unlicensed band. AT\&T Bell Laboratories Technical Journal, pages 118-133, 1997.

[2] John C. Bicket, Bit-Rate Selection in Wireless Networks, MS Thesis, Computer Science and Engineering, MIT, 2005.

[3] A. Vasan, R. Ramjee and T. Woo, "ECHOS - Enhanced Capacity 802.11 Hotspots," in Proc. Of IEEE Infocom, Miami, Florida, USA, 2005.

[4] J. Zhu, X. Guo, L. L. Yang, W. S. Conner, and S. Roy and M. M. Hazra, Adapting physical carrier sensing to maximize spatial reuse in 802.11 mesh networks, Wirel. Commun. Mob. Comput. 2004, vol. 4, pp. 933-946.

[5] J. Zhu, X. Guo, L. Lily Yang, W. Steven Conner, Leveraging spatial reuse in 802.11 mesh networks with enhanced physical carrier sensing, ICC 2004IEEE International Conference on Communications, vol. 27, no. 1, June 2004 pp. 4004-4011

[6] K. Jamieson, B. Hull, A. Miu, and H. Balakrishnan, "Understanding the real-world performance of carrier sense", Procceeding of 2005 ACM SIGCOMM workshop on Experimental approaches to wireless network design and analysis, Philadelphia, Pennsylvania, USA, 2005.

[7] X. Yang, and N. Vaidya, "On Physical Carrier Sensing in Wireless Ad Hoc Networks," in Proc. of IEEE Infocom, Miami, Florida, USA, 2005.

[8] J. Zhu, B. Metzler, X. Guo and Y. Liu, "Adaptive CSMA for Scalable Network Capacity in High-Density WLAN: A hardware Prototyping Approach," in Proc. of IEEE Infocom, Barcelona, Spain, Apr. 2006.

[9] H. Zhai and Y. Fang, Physical Carrier Sensing and Spatial Reuse in Multirate and Multihop Wireless Ad Hoc Networks, IEEE INFOCOM'06.

[10] S. Roy, H. Ma, R. Vijaykumar and J. Zhu, "Optimizing 802.11 Wireless Mesh Network Performance Using Physical Carrier Sensing," UWashington Tech. Report, 2006.

[11] Vivek P. Mhatre, Konstantina Papagiannaki, "Optimal Design of High Density 802.11 WLANs", in Proc. of ACM CoNext 2006.

[12] V. Mhatre, K. Papagiannaki, and F. Baccelli, "Interference Mitigation Through Power Control in High Density 802.11 WLANs," to appear in the proc. of IEEE INFOCOM 2007, Anchorage, Alaska, USA, May 2007.

[13] The Wireless Grid, Whitepaper, Aruba Wireless Networks, http://www.arubanetworks.com 